Research Article

\title{
Attractor and Vector Structure Analyses of Bursting Oscillation with Sliding Bifurcation in Filippov Systems
}

\author{
Rui Qu $(\mathbb{D})$ and Shaolong Li (iD \\ Faculty of Civil Engineering and Mechanics, Jiangsu University, Zhenjiang 212013, China \\ Correspondence should be addressed to Rui Qu; 2111623003@stmail.ujs.edu.cn
}

Received 17 March 2019; Revised 17 May 2019; Accepted 25 June 2019; Published 18 July 2019

Academic Editor: Davood Younesian

Copyright ( $\odot 2019$ Rui Qu and Shaolong Li. This is an open access article distributed under the Creative Commons Attribution License, which permits unrestricted use, distribution, and reproduction in any medium, provided the original work is properly cited.

\begin{abstract}
The main purpose of this paper is to investigate the mechanism of sliding phenomenon in Filippov (nonsmooth) dynamical systems by attractor analysis and vector analysis. A corresponding simple model based on Chua's circuit with periodic excitation was introduced as an example. The attractor analysis proposed in our previous work is used to discuss the complicated oscillations of the Filippov system. However, it failed to perfectly explain the sliding phenomena and establish an analytical method of constant voltage control. Therefore, the geometric structure and analytic conditions of sliding bifurcations in the general $n$ dimensional piecewise smooth system are discussed in detail by vector structure analysis. The prospects of practical application of this method are also discussed in the end.
\end{abstract}

\section{Introduction}

In practical engineering applications, there are many nonsmooth factors, such as switching [1] and impulse control [2]. The nonsmooth models describing the special dynamic behavior are generally divided into three categories: continuous nonsmooth systems [3], Filippov systems [4], and nonsmooth impulsive systems [5]. The Filippov-type (piecewise smooth) systems are characterized by discontinuous vector fields and continuous Jacobian matrices. The introduction of nonsmooth terms will result in a series of special dynamic behaviors near the interface, such as sliding, traversing, grazing, and jumping [6-9]. Due to the discontinuity of the vector field, the traditional attractor analysis method [10] employed to deal with smooth systems cannot be directly used to study the special dynamic behaviors occurring in nonsmooth region. Therefore, new theoretical methods are needed to analyze such problems.

The sliding motions $[11,12]$ with profound physical backgrounds exist widely in engineering practice. For example, in mechanical systems, the introduction of dry friction factor will result in the sliding phenomenon of mechanical oscillator [13]. Similarly, in the circuit system, sliding bifurcation indicates the conservation of voltage, which has certain guiding significance for the design of a constant voltage transformer. Therefore, the analytical conditions of sliding bifurcation [14] are of vital importance.

Taking Chua's circuit system with bilateral diodes as an example, the bursting oscillation and sliding bifurcation are studied by equilibrium curve, bifurcation diagram, and phase portrait. The validity of the theoretical results is verified by calculation of the sliding time and analysis of simulation data near special points. The application prospects of methods proposed are discussed in the end.

\section{Mathematical Model}

The current-driven Chua's circuit is shown in the Figure 1. The circuit consists of capacitors $C_{1}$ and $C_{2}$, inductor $L$, resistors $R$ and $R_{\mathrm{N}}$, and current source $i_{\mathrm{s}}$, in which $R_{\mathrm{N}}$ is a nonlinear resistor. According to Kirchhoff's current law and voltage law, the state equations of the circuit can be formed as 


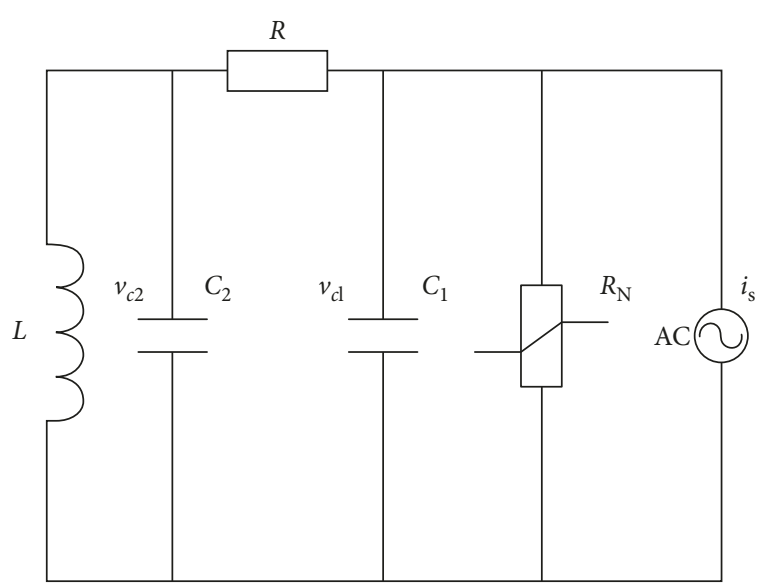

FIgURE 1: The current-driven Chua's circuit.

$$
\left\{\begin{array}{l}
\frac{d v_{c 1}}{d t}=\frac{1}{R C_{1}}\left(v_{c 1}-v_{c 2}\right)-\frac{1}{C_{1}} f\left(v_{c 1}\right)+\frac{1}{C_{1}} i_{s} \\
\frac{d v_{c 2}}{d t}=\frac{1}{R C_{2}}\left(v_{c 1}-v_{c 2}\right)+\frac{1}{C_{1}} i_{1} \\
\frac{d i_{1}}{d t}=-\frac{1}{L} v_{c 2},
\end{array}\right.
$$

where $v_{c 1}$ and $v_{c 2}$ are the voltages of $C_{1}$ and $C_{2}$, respectively; $i_{1}$ is the current of $L$; and $f\left(v_{c 1}\right)$ is the volt-ampere characteristic function of a nonlinear resistor. The excitation current $[15,16]$ is denoted by $i_{\mathrm{s}}=F \sin (\Omega t+\Phi)$ in which $F, \Omega$, and $\Phi$ represent the amplitude, frequency, and initial phase of the excitation current, respectively. By the coordinate and dimensionless transformation,

$$
\begin{aligned}
& x=v_{c 1}, \\
& y=v_{c 2}, \\
& z=R i_{1}, \\
& t=R C_{2} \tau, \\
& \alpha=\frac{C_{2}}{C_{1}}, \\
& \beta=\frac{R^{2} C_{2}}{L}, \\
& A=\frac{R C_{2} F}{C_{1}}, \\
& \omega=R C_{2} \Omega .
\end{aligned}
$$

The state equations of Chua's circuit excited by current can be expressed as

$$
\left\{\begin{array}{l}
\dot{x}=\alpha(y-x-f(x))+A \sin (\omega t), \\
\dot{y}=x-y+z, \\
\dot{z}=-\beta y .
\end{array}\right.
$$

where the volt-ampere characteristic function $f(x)$ of the nonlinear resistance can be expressed in the form of cubic polynomial proposed by Hartley and Mossayebi [17] in the study of deformed Chua's circuit as $f(x)=m x+n x^{3}$, where $m=-(8 / 7)$ and $n=2 / 7$. It is noteworthy that the nonlinearity is weak due to the relatively small nonlinear coefficient.

Taking the simple Chua's circuit system as an example, dynamic behaviors of Filippov systems with multiscale effect can be studied. By adding a bilateral diode as a nonlinear element, the dimensionless mathematical model of the modified circuit can be expressed as follows:

$$
\left\{\begin{array}{l}
\dot{x}=\alpha\left(y+a x^{3}+b x\right)+w+c \operatorname{sgn}(x), \\
\dot{y}=x-y+z, \\
\dot{z}=-\beta y
\end{array}\right.
$$

where $w=A \sin (\Omega t)$ is the external excitation, and when the frequency of external excitation is far less than the natural frequency $\omega$, there will be two time scale effects. Nonsmooth interface $x=0$ divides the original system into two subsystems located in different phase spaces.

\section{Bifurcation Analysis of Two Subsystems}

There are magnitude differences between external excitation frequency and natural frequency when $0<\Omega \ll 1$. In any natural frequency period $T_{\omega}=2 \pi / \omega, t \in\left[t_{0}, t_{0}+T_{\omega}\right]$, the external excitation will change between $W_{A}=A \sin \left(\Omega t_{0}\right)$ and $W_{B}=A \sin \left(\Omega t_{0}+(2 \pi \Omega / \omega)\right)$. Because $\Omega / \omega \ll 1, W_{A} \approx$ $W_{B}$ which means that in any natural frequency period, the external excitation is almost a constant. Therefore, the whole external excitation term can be regarded as a slowly varying parameter and two generalized autonomous subsystems can be derived as follows.

When $x<0$,

$$
S_{1}:\left\{\begin{array}{l}
\dot{x}=\alpha\left(y+a x^{3}+b x\right)+w-c, \\
\dot{y}=x-y+z, \\
\dot{z}=-\beta y .
\end{array}\right.
$$

When $x>0$,

$$
S_{2}:\left\{\begin{array}{l}
\dot{x}=\alpha\left(y+a x^{3}+b x\right)+w+c, \\
\dot{y}=x-y+z, \\
\dot{z}=-\beta y .
\end{array}\right.
$$

The nonsmooth interface $\Sigma(\Sigma:(x, y, z) \mid x=0)$ divides the phase space into two regions. The corresponding regions of $x>0$ and $x<0$ are represented as $D_{+}$and $D_{-}$, respectively. When the trajectory is located in $D_{-}$, it is restricted by subsystem $S_{1}$. Once the trajectory reaches $D_{+}$through the 
interface, the governing equation will be converted to $S_{2}$ immediately. The equilibrium point of two subsystems can be expressed as $E_{0}(x, y, z)=\left(X_{0}, 0,-X_{0}\right)$, where $X_{0}$ satisfying

$$
\alpha\left(a X_{0}^{3}+b X_{0}\right)+w+c \operatorname{sgn}\left(X_{0}\right)=0 .
$$

The properties of the equilibrium point depend on the following characteristic equation:

$$
\begin{aligned}
& \lambda^{3}+\left(1-\alpha b-3 \alpha a X_{0}^{2}\right) \lambda^{2}+\left(\beta-\alpha-\alpha b-3 \alpha a X_{0}^{2}\right) \\
& -\alpha \beta\left(b+3 a X_{0}^{2}\right)=0
\end{aligned}
$$

Therefore, the conditions for the stability of equilibrium point $E_{0}$ are as follows:

$$
\begin{aligned}
& 1-\alpha b-3 \alpha a X_{0}^{2}>0 \\
& \alpha \beta\left(b+3 a X_{0}^{2}\right)<0 \\
& \left(1-\alpha b-3 \alpha a X_{0}^{2}\right)\left(\beta-\alpha-\alpha b-3 \alpha a X_{0}^{2}\right) \\
& \quad+\alpha \beta\left(b+3 a X_{0}^{2}\right)>0
\end{aligned}
$$

Two types of codimension-one bifurcations can be observed, in which fold bifurcations occur under the following conditions:

$$
\mathrm{FB}:\left\{\begin{array}{l}
b+3 a X_{0}^{2}=0, \\
\alpha\left(a X_{0}^{3}+b X_{0}\right)+w+c \operatorname{sgn}\left(X_{0}\right)=0 .
\end{array}\right.
$$

With $1-\alpha b-3 \alpha a X_{0}^{2}>0,\left(1-\alpha b-3 \alpha a X_{0}^{2}\right)(\beta-\alpha-\alpha b-$ $\left.3 \alpha a X_{0}^{3}\right)+\alpha \beta\left(b+3 a X_{0}^{2}\right)>0$.

Based on the Routh-Hurwitz stability criterion [2, 3], for a cubic polynomial,

$$
P_{3}(z)=z^{3}+a_{1} z^{2}+a_{2} z^{3}+a_{3} .
$$

Its Routh-Hurwitz conditions can be formed as

$$
\begin{aligned}
& a_{1}>0,\left|\begin{array}{ll}
a_{1} & 1 \\
a_{3} & a_{2}
\end{array}\right|>0, a_{3}\left(a_{1} a_{2}-a_{3}\right)>0, \\
& \mathbb{I} \\
& a_{1}>0, a_{3}>0, a_{1} a_{2}>a_{3} .
\end{aligned}
$$

When condition $a_{1} a_{2}>a_{3}$ degenerates to $a_{1} a_{2}=a_{3}$, a pair of pure imaginary roots appear in the solution of characteristic polynomial (8) indicating that Hopf bifurcation occurs. Therefore the conditions of Hopf bifurcation are as follows:

$$
\mathrm{HB}:\left\{\begin{array}{l}
\left(1-\alpha b-3 \alpha a X_{0}^{2}\right)\left(\beta-\alpha-\alpha b-3 \alpha a X_{0}^{2}\right) \\
+\alpha \beta\left(b+3 a X_{0}^{2}\right)=0, \\
\alpha\left(a X_{0}^{3}+b X_{0}\right)+w+c \operatorname{sgn}\left(X_{0}\right)=0 .
\end{array}\right.
$$

With $1-\alpha b-3 \alpha a X_{0}^{2}>0, \alpha \beta\left(b+3 a X_{0}^{2}\right)<0$.

The bifurcation and bursting oscillation behaviors of this system have been studied in our previous work [14]. Therefore, No further elaboration will be made here. The two-parameter bifurcation diagram and the equilibrium branches with bifurcations are shown in Figure 2 as a supplement to our previous work.
In Figure 2, $\mathrm{FB} i \pm$ and $\mathrm{HB} i \pm(i=1,2)$ corresponds to the different bifurcation points in different segments of equilibrium branches, respectively. The bifurcation lines divided the parametric plane into different regions marked by number $i(i=1,2, \ldots, 7)$, and the stability of the equilibrium points corresponding to the adjacent regions is opposite. The results of Figure 2 are consistent with those of our previous study.

\section{Attractor Structure Analysis of Sliding Motion}

It is noteworthy that all the bifurcation results obtained are related to slow-varying parameters [18-22]. Therefore, in order to reveal the complete bursting oscillation process and special dynamic behaviors of the system (4), the concept of transformed phase portrait (TPP) [23, 24] should be introduced.

4.1. TPP for the Trajectory $\Pi_{T}:\{[x(t), y(t), z(t)], t \in R\}$. The traditional phase portrait reflects the relationship among state variables in the phase space $(x, y, z)$ or the projection planes $(x, y)$ and $(y, z)$. If the slowly varying parameter is regarded as a generalized state variable, the generalized phase trajectory can be obtained $\Pi_{\mathrm{GT}}:\{[x(t)$, $y(t), z(t), w(t), t \in R]\}$, where $w(t)=A \sin (\Omega t)$. The generalized phase trajectories in the generalized phase space $(x, y, z, w)$ and its projected phase plane are called transformed phase portraits, which are used to characterize the relationship between different state variables and slowly varying parameters.

Variable parameters $b$ were taken $b=0.14$ and $b=0.70$, respectively, while other parameters were fixed as

$$
\begin{aligned}
& \alpha=6.9, \\
& \beta=10.0, \\
& a=-\frac{2}{7} \\
& c=-2, \\
& A=6.0 .
\end{aligned}
$$

Therefore, $w$ varies periodically with frequency 0.01 in the interval $[-6.0,6.0]$. Taking $A=0$, the natural frequency of the system can be calculated. For $b=0.14$, the equilibrium point of the two subsystems is approximately $E_{ \pm}=( \pm 1.1662,0.0, \mp 1.1662)$, with its corresponding eigenvalue as follows:

$$
\begin{aligned}
& \lambda_{1}=-7.920, \\
& \lambda_{ \pm}=-0.0784 \pm 2.988 I,
\end{aligned}
$$

indicating that the natural frequency is about 2.988. Similarly, when $b=0.70$, the coordinates of the three equilibrium points and their corresponding eigenvalues are 


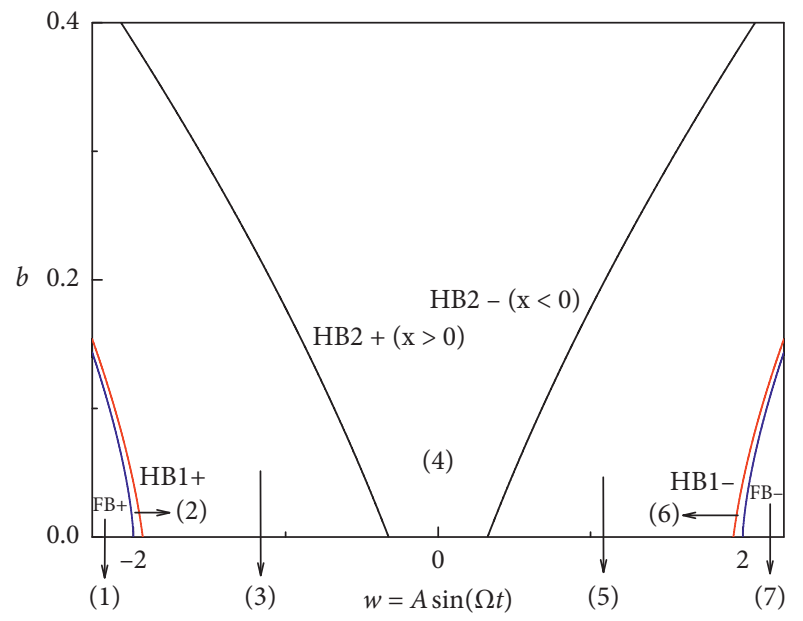

(a)

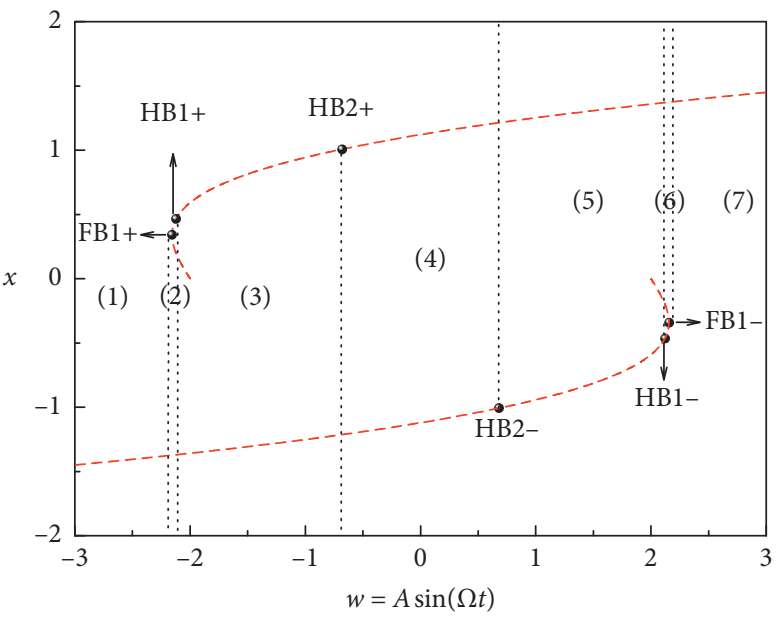

(b)

Figure 2: (a) Two-parameter bifurcation diagram and (b) the equilibrium branches with bifurcations in different regions.

$$
\begin{aligned}
E_{1 \pm} & =( \pm 0.4517,0.0, \mp 0.4517), \\
\lambda_{1} & =4.5159, \\
\lambda_{ \pm} & =-0.9463 \pm 2.6698 I, \\
E_{2 \pm} & =( \pm 1.2897,0.0, \mp 1.2897), \\
\lambda_{1} & =-6.0386, \\
\lambda_{ \pm} & =0.01534 \pm 2.8797 I, \\
E_{3 \pm} & =( \pm 1.7414,0.0, \mp 1.7417), \\
\lambda_{1} & =-13.622, \\
\lambda_{ \pm} & =-0.2417 \pm 3.0923 I .
\end{aligned}
$$

Therefore, when the trajectory oscillates near different equilibrium points $E_{j \pm}(j=1,2,3)$, its natural frequencies are about 2.6698, 2.8797, and 3.0923.

4.2. Scenario 1: $b=0.14$. The $3 \mathrm{D}$ phase portrait and bifurcation diagram with parameter $b$ are shown in Figure 3. It can be seen that the attractor structure keeps the symmetry of the original system. The alternation of large-amplitude spiking states and small-amplitude quiescent states can be observed. Specially, the sliding phenomenon appears on the nonsmooth boundary indicating that the voltage of the capacitor remained a constant during this period of time. It should be pointed out that the phenomenon in Figure 3(b) does not mean the changes of periodicity (similar to period doubling bifurcation) but that the position of equilibrium points migrates due to the occurrence of sliding bifurcation which will be discussed in detail.

In order to reveal the mechanism of bursting oscillation, the overlap of the transformed phase portrait [14] with the equilibrium branches can be observed in Figure 4. $E B_{j}^{(i)}(i=$ $1,2 ; j=1,2, \ldots, 6)$ corresponds to the equilibrium curve segments of subsystem $S_{i}(i=1,2)$, respectively. The solid part of the equilibrium line represents the stable equilibrium state, and the dashed line represents the unstable state. The black lines represent the equilibrium points that can be found in the corresponding region, while the red parts denote the equilibrium state that exists but is not located in the phase space of the corresponding system in control.

4.2.1. Bursting Oscillation Mechanism. As shown in Figure 4, the trajectory dominated by subsystem $S_{1}$ starts from point $A_{1}(w=-6.0)$ and runs almost strictly along its stable equilibrium state $\mathrm{EB}_{1}^{(1)}$. Despite the existence of a stable limit cycle $\mathrm{LC}_{1}^{(1)}$ between the two supercritical Hopf bifurcation points $\mathrm{HB}_{1}^{(1)}$ and $\mathrm{HB}_{2}^{(1)}$, the trajectory still runs along the stable equilibrium, showing the quiescent state. The stability of limit cycles produced by these two Hopf bifurcations is opposite. They disappear after contact suggesting the occurrence of LPC bifurcation. When trajectory reaches the fold bifurcation point $\mathrm{FB}_{1}^{(1)}$, it jumps directly to the stable equilibrium. As time $t$ continues to increase, the trajectory is attracted by the only stable equilibrium segment $\mathrm{EB}_{6}^{(1)}$ of subsystem $S_{1}$ until it intersects at $A_{2}$ with the nonsmooth boundary $\Sigma$.

4.2.2. Supplementary Notes. It should be pointed out that although there are Hopf bifurcation points $\mathrm{HB}_{1}^{(1)}$ and $\mathrm{HB}_{2}^{(1)}$ on the equilibrium as shown in Figure 4, the trajectory still runs along the equilibrium due to the slow path effect which causes the bifurcation lag [25]. The phenomenon that the trajectory contacts the nonsmooth boundary and runs along $\Sigma$ is called sliding motion. The sliding process is still in quiescent state until it reaches point $A_{3}$. Besides, it can be clearly seen from Figure 3(a) that when trajectory is located on the nonsmooth boundary (sliding motions), the original system will degenerate into a two-dimensional system:

$$
\left\{\begin{array}{l}
\dot{y}=x-y+z, \\
\dot{z}=-\beta y
\end{array}\right.
$$

indicating that there will be a new equilibrium point (stable focus equilibrium point) on the interface $x=0$, named 


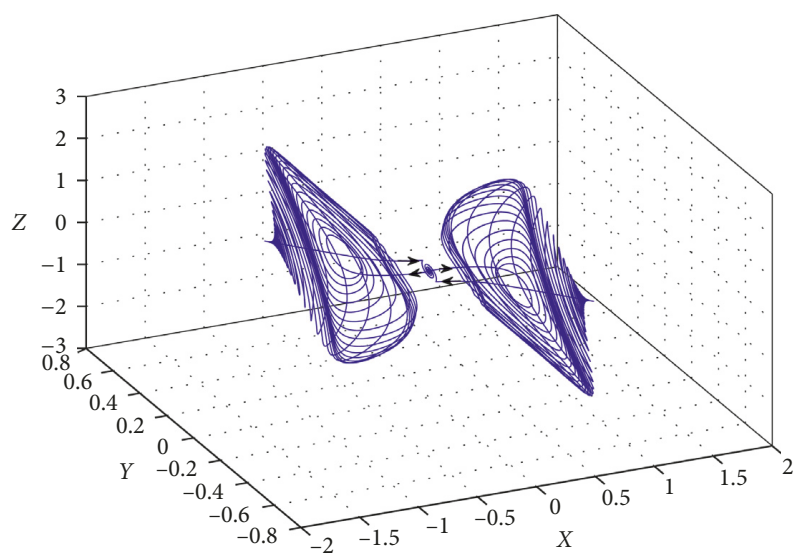

(a)

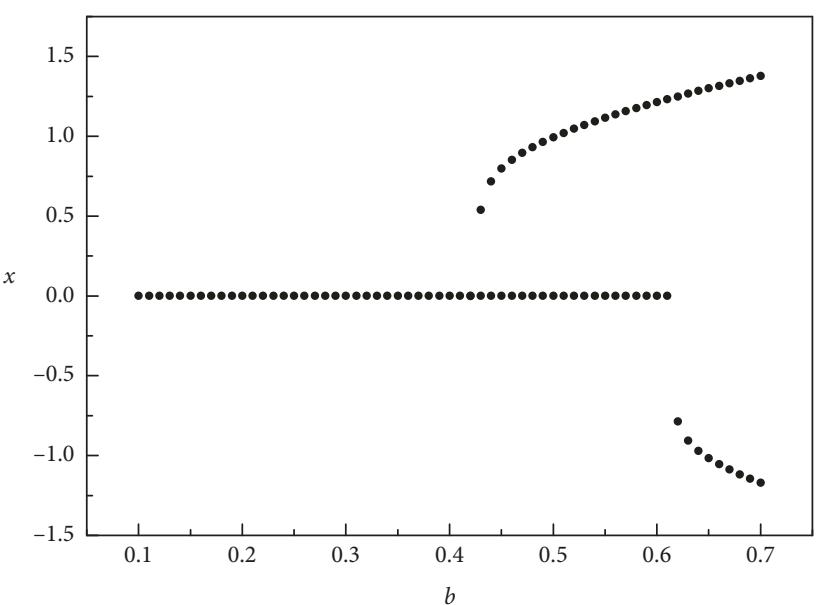

(b)

FIgURE 3: (a) 3D phase portrait with $b=0.14$ and (b) bifurcation diagram with parameter $b$.

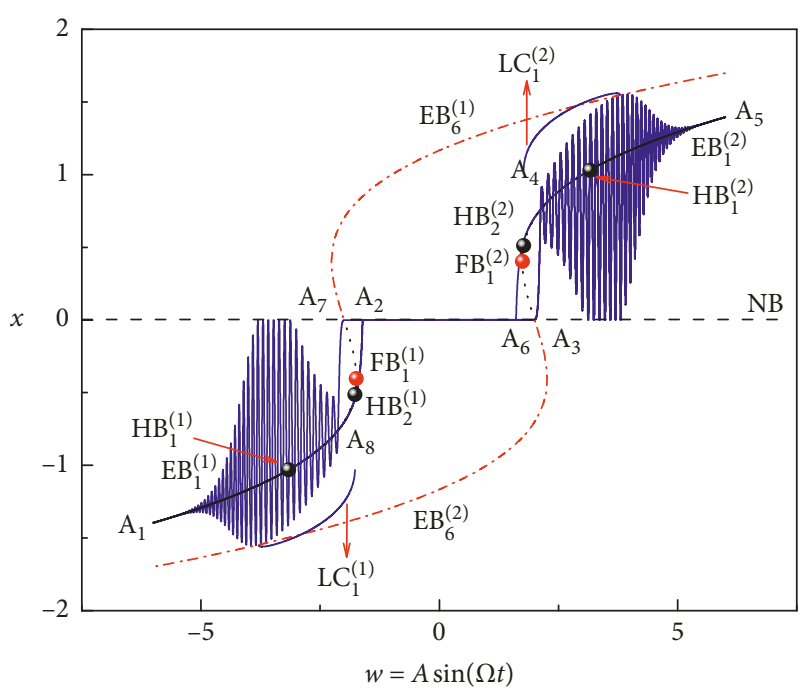

FIGURE 4: Overlap of the transformed phase portrait with the equilibrium branches.

pseudoequilibrium point. With the variation of parameter $\beta=R^{2} C_{2} / L$, the pseudoequilibrium will also bifurcate.

\subsubsection{Attractor Structure When the Sliding Phenomenon} Occurs. When the phase trajectory approaches the boundary from side $D_{-}$, it is attracted by $\mathrm{EB}_{6}^{(1)}$ located in $D_{+}$on subsystem $S_{1}$, so it has the tendency to move across the boundary to $D_{+}$. However, once the trajectory reaches $D_{+}$, the control system will be switched to $S_{2}$ immediately. The corresponding stable equilibrium $\mathrm{EB}_{6}^{(2)}$ is located at $D_{-}$, which makes the trajectory return to $D_{-}$. The alternating control of two subsystems is the intrinsic cause of the sliding phenomenon.

4.2.4. Sliding Time Calculation. In our previous work [14], the sliding time has been estimated by the time required from $\mathrm{FB}_{1}^{(1)}$ to $A_{3}$ with insufficient accuracy. In addition, this estimation method is not universal. New method are ought to be proposed, which will be discussed in the next section.

4.2.5. Discussion on Physical Meaning. In general, fold, Hopf, and sliding bifurcation, respectively, correspond to mutation, oscillation, and conservation of circuit component voltage in the circuit system. A constant voltage transformer is widely used in engineering practice. The above results show that constant voltage control can be achieved by choosing appropriate nonlinear resistors and the output time of constant voltage can accurately be controlled by the calculation method of sliding time. The key point is how to deduce the analytical expression of sliding motions (constant voltage) which will be discussed in Section 5.

4.3. Scenario $2: b=0.70$. In this case, a problem which was not well resolved in [14] should be pointed out. The overlap of the transformed phase portrait with the equilibrium branches is shown in Figure 5(a), and the local enlargement of time history is shown in Figure 5(b).

Although the distribution of attractor structure in case 2 is different from that in case 1 , most of its dynamic behavior can be explained on the basis of previous studies, so it is not necessary to elaborate on this for article length consideration. It is noteworthy that as shown in local enlargement at the lower right corner of Figure 5(a), when the trajectory slides from point $A_{2}$, a small jump occurs immediately and then returns to the interface to continue sliding. This seemingly insignificant phenomenon cannot be well explained by attractor structure analysis due to the coexistence of multiple equilibrium points. In order to further study the special dynamic behavior of nonsmooth systems at nonsmooth boundary, it is obviously not enough to use attractor structure analysis alone. Therefore, based on differential inclusion theory and focusing on the local dynamics near the interface, another method to analyze such problems named vector structure analysis is proposed. 


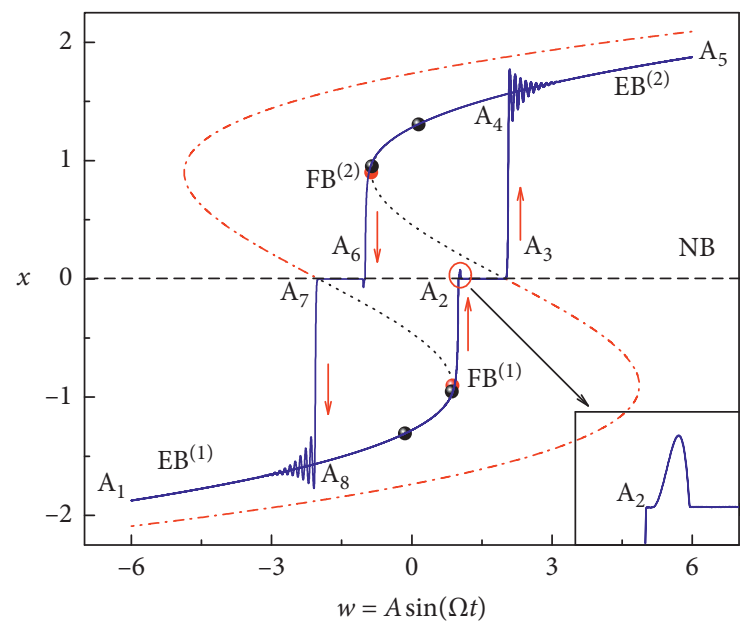

(a)

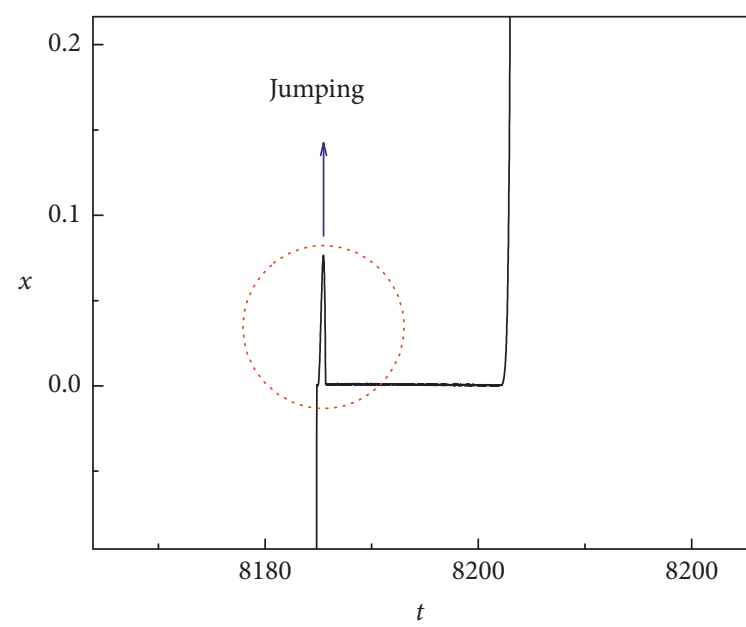

(b)

FIGURE 5: (a) Overlap of the transformed phase portrait with the equilibrium branches and (b) local enlargement of time history.

\section{Structural Characteristics of Vector Field for Sliding Bifurcation}

Consider a general $n$-dimensional piecewise smooth system:

$$
\dot{Y}= \begin{cases}F_{1}(Y), & h(Y)>0, \\ F_{2}(Y), & h(Y)<0 .\end{cases}
$$

The nonsmooth boundary can be defined as $\Sigma=$ $\left\{Y \in R^{n+1} \mid h(Y)=0\right\}$. The vector field of the sliding region can be represented by convex closures of $F_{1}(Y)$ and $F_{2}(Y)$ :

$$
F_{s}(Y)=\frac{F_{1}(Y)+F_{2}(Y)}{2}+\frac{F_{1}(Y)-F_{2}(Y)}{2} \alpha(Y)
$$

where $-1 \leq \alpha(Y) \leq 1$. Assuming that $h_{Y}=\left(\partial h / \partial y_{1}\right.$, $\left.\partial h / \partial y_{2}, \ldots\right)^{T}=\nabla h$, so $h_{Y} F_{S}(Y)=0$ (normal vector $h_{Y}$ is perpendicular to the nonsmooth interface, while $h_{Y} F_{S}(Y)=0$ is parallel to the interface). Therefore, $\alpha(Y)$ can be expressed as

$$
\alpha(Y)=-\frac{h_{Y}\left(F_{1}+F_{2}\right)}{h_{Y}\left(F_{2}-F_{1}\right)} .
$$

The sliding region and its boundary can be expressed as

$$
\begin{aligned}
\widehat{\Sigma} & =\{Y \in \Sigma \mid-1 \leq \alpha(Y) \leq 1\}, \\
\partial \widehat{\Sigma}^{ \pm} & =\{Y \in \Sigma \mid \alpha(Y)= \pm 1\},
\end{aligned}
$$

where $\partial \widehat{\Sigma}^{-}$is the tangent point of $F_{1}(Y)$ on nonsmooth boundary $\Sigma$. So, $h_{Y} F_{1}(Y)=0$ and $h_{Y} F_{2}(Y)=0$. By deriving the two sides of formula (20) separately,

$$
\begin{aligned}
\alpha_{Y}= & -\frac{\left(h_{Y}\left(F_{1}+F_{2}\right)\right)_{Y}\left(h_{Y}\left(F_{2}-F_{1}\right)\right)}{\left(h_{Y}\left(F_{2}-F_{1}\right)^{2}\right)} \\
& +\frac{\left(h_{Y}\left(F_{1}+F_{2}\right)\right)\left(h_{Y}\left(F_{2}-F_{1}\right)\right)_{Y}}{\left(h_{Y}\left(F_{2}-F_{1}\right)^{2}\right)} \\
= & -\frac{\left(h_{Y} F_{1 Y}+h_{Y Y} F_{1}+h_{Y} F_{2 Y}+h_{Y Y} F_{2}\right)\left(h_{Y} F_{2}-h_{Y} F_{1}\right)}{\left(h_{Y} F_{2}-h_{Y} F_{1}\right)^{2}} \\
& +\frac{\left(h_{Y} F_{2 Y}+h_{Y Y} F_{2}-h_{Y} F_{1 Y}-h_{Y Y} F_{1}\right)\left(h_{Y} F_{1}+h_{Y} F_{2}\right)}{\left(h_{Y} F_{2}-h_{Y} F_{1}\right)^{2}} \\
= & -\frac{\left(h_{Y} F_{1 Y}+h_{Y} F_{2 Y}\right)\left(h_{Y} F_{2}-h_{Y} F_{1}\right)}{\left(h_{Y} F_{2}-h_{Y} F_{1}\right)^{2}} \\
& +\frac{\left(h_{Y} F_{2 Y}-h_{Y} F_{1 Y}\right)\left(h_{Y} F_{1}+h_{Y} F_{2}\right)}{\left(h_{Y} F_{2}-h_{Y} F_{1}\right)^{2}},
\end{aligned}
$$

$\partial \widehat{\Sigma}^{-}$satisfying $F_{\mathrm{S}}(Y)=F_{1}(Y)$, indicating that $h_{Y} F_{1}=0$. Combining with (23), normal vectors on $\partial \widehat{\Sigma}^{-}$can be expressed as

$$
\alpha_{Y}=-\frac{2}{h_{Y} F_{2}} h_{Y} F_{1 Y} .
$$

The physical meaning of differential inclusion theory can be understood simply through dry friction: sliding friction corresponds to the boundary of sliding region, static friction corresponds to the interior of sliding region, and the numerical value of static friction depends on the equilibrium condition. 
5.1. Geometric Classification of Sliding Bifurcations. According to the geometric structure of the periodic solution trajectory intersecting with the sliding region and its boundary, the sliding bifurcation can be roughly divided into the following four categories, as shown in Figure 6.

The characteristic of crossing sliding bifurcation is that the trajectory crosses the boundary of the sliding region at the bifurcation point and leaves (as shown in Figure 6(a) trajectory b). Then the sliding phenomena occur when the contact points enter the sliding area with the change of parameters (as shown in the trajectory c of Figure 6(a)).

The grazing sliding bifurcation, as the name implies, grazes along the boundary of the sliding region at the bifurcation point and leaves (as shown in Figure 6(b) track b). Then, the sliding phenomena occur when the contact points enter the sliding area with the change of parameters (as shown in the trajectory c of Figure 6(b)).

The switching sliding bifurcation is similar to crossing sliding bifurcation, but the difference is that a section of track is still in the sliding region after traversing the boundary of the sliding region at the bifurcation point (as shown in Figure 6(c) trajectory $b$ ). Then, a segment of the trajectory remains in the sliding region as the parameters change (as shown in Figure 6(c) trajectory c).

Unlike the other three bifurcations, the bifurcated trajectories of the adding sliding bifurcation are completely located in the sliding region and tangent to the boundary of the sliding region at the bifurcation point (as shown in track b of Figure $6(d)$ ). As the parameters change, a section of the track is still located in the sliding region (as shown in track $\mathrm{c}$ of Figure 6(d)).

5.2. Analytical Conditions for Sliding Bifurcation. The above four kinds of sliding bifurcations can accurately be described by analytic conditions.

5.2.1. Crossing Sliding Bifurcation and Grazing Sliding Bifurcation. Because the bifurcation point $Y^{*}$ is located on the nonsmooth boundary,

$$
h\left(Y^{*}\right)=0, h_{Y}\left(Y^{*}\right) \neq 0 .
$$

Due to $Y^{*} \in \partial \widehat{\Sigma}^{-}$,

$$
\left.\frac{d}{d t} h\left(\phi\left(Y^{*}, t\right)\right)\right|_{t=0}=h_{Y} F_{1}\left(Y^{*}\right)=0
$$

where $\phi$ is the corresponding smooth solution manifold. In addition, after crossing the interface, the trajectory moves away from the sliding area, so

$$
\left.\frac{d}{d t} \alpha\left(\phi\left(Y^{*}, t\right)\right)\right|_{t=0}=\alpha_{Y} F_{1}\left(Y^{*}\right)<0 \text {. }
$$

By the law of derivation of the dot product and the combination of formulas (24), (26), and (27),

$$
\left.\frac{d^{2}}{d t^{2}} h\left(\phi\left(Y^{*}, t\right)\right)\right|_{t=0}=h_{Y} F_{1 Y} F_{1}\left(Y^{*}\right)>0 \text {. }
$$

5.2.2. Switching Sliding Bifurcation. This situation also satisfies the two basic conditions (25) and (26), but the track crosses the sliding area at the bifurcation point and remains in the sliding area; therefore,

$$
\begin{aligned}
& \left.\frac{d}{d t} \alpha\left(\phi\left(Y^{*}, t\right)\right)\right|_{t=0}=\alpha_{Y} F_{1}\left(Y^{*}\right)>0, \\
& \left.\frac{d^{2}}{d t^{2}} h\left(\phi\left(Y^{*}, t\right)\right)\right|_{t=0}=h_{Y} F_{1 Y} F_{1}\left(Y^{*}\right)<0 .
\end{aligned}
$$

5.2.3. Adding Sliding Bifurcation. The two basic conditions (25) and (26) are still valid, but in this case, the bifurcation trajectory is completely located within the sliding region and tangent to the boundary of the sliding region at the bifurcation point, so the conditions (28) and (30) for the above three cases degenerate:

$$
\left.\frac{d^{2}}{d t^{2}} h\left(\phi\left(Y^{*}, t\right)\right)\right|_{t=0}=h_{Y} F_{1 Y} F_{1}\left(Y^{*}\right)=0 .
$$

Because the bifurcation trajectory has a minimum value at $\partial \widehat{\Sigma}^{-}$, there is a nondegeneration condition:

$$
\left.\frac{d^{2}}{d t^{2}} \alpha\left(\phi\left(Y^{*}, t\right)\right)\right|_{t=0}=\alpha_{Y} F_{1 Y} F_{1}\left(Y^{*}\right)>0 .
$$

By the law of derivation of the dot product and the combination of formulas (24), (26), and (32),

$$
\left.\frac{d^{3}}{d t^{3}} h\left(\phi\left(Y^{*}, t\right)\right)\right|_{t=0}=h_{Y}\left(F_{1 Y}\right)^{2} F_{1}\left(Y^{*}\right)<0 .
$$

5.3. Vector Field Structure Characteristics of Sliding Bifurcation. Based on the previous analysis, the vector field structure of sliding bifurcation can be summarized.

Regardless of the type of sliding bifurcation, as long as the trajectory is located in the sliding region, its vector field must have the structural characteristics as shown in Figure 7(a).

Sliding phenomena occur when there are opposite vector fields on both sides of the nonsmooth interface. The direct reason is that the trajectory is forced by the opposite vector field near the boundary and thus can only oscillate along the boundary. When the limit of step size approaches zero, it is a strict sliding motion. With opposite vector field in Figure 7(a), if trajectory locates in $D_{-}$, it will be pushed to nonsmooth boundary. For the same reason, if it locates in $D_{+}$, it will also approach the boundary. Therefore, it can be understood as that the trajectory is squeezed by the vector field and can only strictly slide along the nonsmooth boundary. This result is consistent with the conclusions of attractor structure analysis. In contrast, when vector fields with the same sign exist on both sides of the nonsmooth boundary, the trajectory traverses the interface directly, as shown in Figure 7(b).

In scenario 2, the transient jumping phenomenon after sliding is caused by the change of external excitation in the sliding region, which leads to the change of vector field, until the vector field on one side weakens gradually and eventually reverses. It should be pointed out that although there is only one stable attractor on both sides of the interface, the 


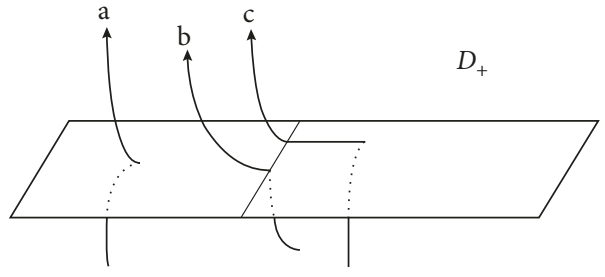

$D_{-}$

(a)

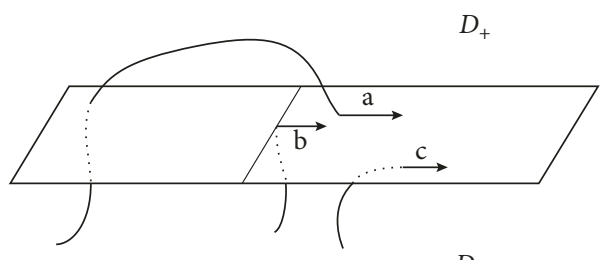

$D_{-}$

(c)

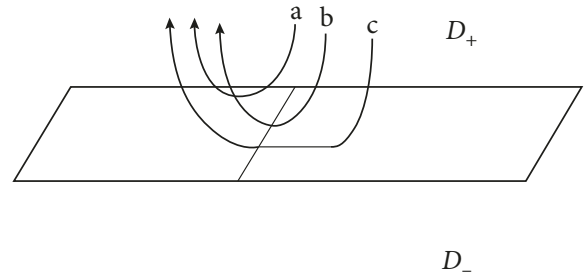

(b)

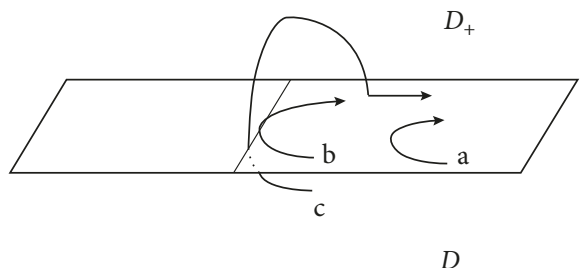

(d)

Figure 6: (a) Crossing, (b) grazing, (c) switching, and (d) adding sliding bifurcations.

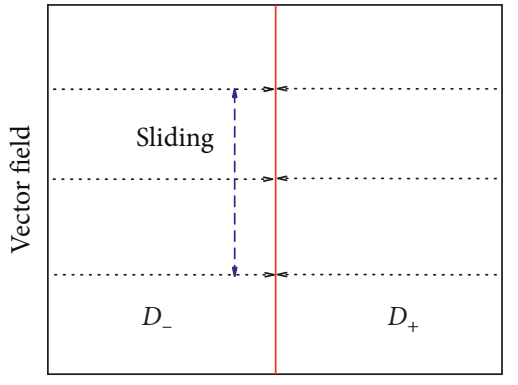

Nonsmooth boundary

(a)

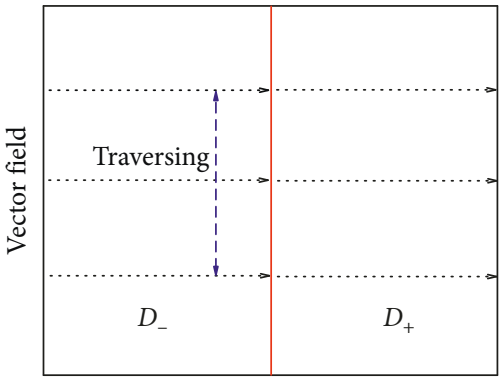

Nonsmooth boundary

(b)

FiguRE 7: The structure of the vector field in (a) sliding region and (b) traversing region.

complete sliding phenomenon may not occur. This is because the stability of the attractor does not directly determine the direction of the normal component of the vector field at the interface, as shown in diagrammatic sketch Figure 8.

For instance, supposing that subsystem defined in $D_{+}$ has a stable focus (or limit cycle) $P_{+}$in $D_{-}$. The direction of the vector field in its domain of attraction is shown by the blue arrows. It can be clearly seen that although $P_{+}$is attractive to the phase trajectory in $D_{+}$in the long run, its vector field on the side of $D_{+}$does not point to the nonsmooth boundary (as shown in the dotted part). Therefore, although the attractor structure analysis methods (such as fast-slow analysis method) have some advantages in studying the global dynamic behaviors of nonsmooth periodic excitation system, it also has some limitations in dealing with some special local dynamic details such as sliding bifurcation.

5.4. Numerical Verification. In order to better understand the mechanism of sliding bifurcation, the sliding process in case 2 is numerically analyzed as Table 1.
It is noteworthy that in sliding region $A_{2} \longrightarrow A_{3}$, the signs of vector field $\dot{x}$ on both sides of nonsmooth boundary $x=0$ are opposite and the difference is exactly equal to $2|c|=$ $2 \times 2.0=4.0$ ( $c$ is the coefficient of the symbolic function shown in formula (14)). Based on the analytical expressions (20)-(22) of the sliding region of the upper section, it can be found that the numerical results are in good agreement with the theoretical results.

5.5. Discussion on Physical Meaning. The proposed analytical conditions of sliding bifurcation imply that the constant voltage can be controlled by changing the external excitation (AC source) rather than the properties of the circuit elements themselves. Besides, the sliding phenomenon also exists in mechanical systems such as spring oscillator considering dry friction factor, etc. Actually, in engineering practice, the sliding (sticking) phenomenon is not always expected. For instance, the occurrence of sliding phenomenon of the lathes for producing models with spring oscillator means the production of defective products. Therefore, finding out a way to eliminate the sliding motions 


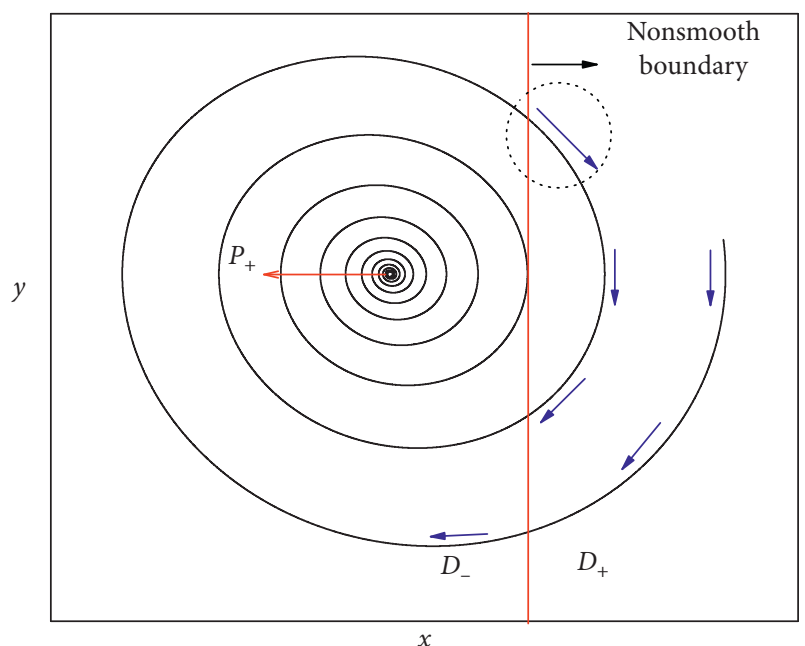

Figure 8: The vector field of a stable focus.

TABLE 1: Simulation data near points of special behaviors.

\begin{tabular}{lcccc}
\hline & $w$ & $x$ & $y$ & $\dot{x}$ \\
\hline \multirow{2}{*}{$A_{2}$ (jumping) } & 1.0085 & $\approx 0,>0$ & 0.1413 & $\approx 0,<0$ \\
$\ldots$ & 1.0091 & $\approx 0,>0$ & 0.1436 & $\approx 0,>0$ \\
$\ldots$ & $\ldots$ & $\ldots$ & $\ldots$ & $\ldots<0$ \\
$A_{2} \longrightarrow A_{3}$ (sliding) & 1.5001 & $\approx 0,>0$ & 0.00285 & $-0.4769<0$ \\
& 1.5006 & $\approx 0,<0$ & 0.00281 & $+3.5230>0$ \\
\hline
\end{tabular}

is also of great concern. Controlling the frequency of external excitation instead of changing the property of the material itself is a relatively simple and effective solution because the change of external excitation invalidates the necessary condition of sliding bifurcation mentioned in the manuscript. This part of the content will be further explored in our next work.

\section{Conclusions}

The introduction of bidirectional diodes in the circuit system will result in the occurrence of sliding bifurcation indicating the conservation of voltage. The selection of different nonlinear resistors corresponds to different sliding time and modes. Sliding motions can be divided into four categories according to the corresponding geometric characteristics. The specific expression of sliding area and its boundary along with the special structure of vector field are presented. Vector fields on both sides of nonsmooth boundary are opposite in sliding region while remain the same in traversing region.

In addition, this paper mainly focuses on the single variable of nonlinear resistance. Coupling effects between multiple factors will be discussed separately in other literature.

\section{Data Availability}

All data generated or analyzed during this study are included in this published article. Requests for material should be made to the corresponding author.

\section{Conflicts of Interest}

The authors declare that there are no conflicts of interest regarding the publication of this article.

\section{Acknowledgments}

This work was supported by the National Natural Science Foundation of China (grant no. 11632008).

\section{References}

[1] N. Berglund and D. London, "Mixed-mode oscillations and interspike interval statistics in the stochastic FitzHughNagumo model," Nonlinearity, vol. 25, no. 8, pp. 2302-2335, 2012.

[2] J. Hale, Functional Differential Equations, vol. 3, SpringerVerlag, New York, NY, USA, 1971.

[3] A. K. Yuri, Elements of Applied Bifurcation Theory, SpringerVerlag, New York, NY, USA, 1995.

[4] Z. Shang, J. Jiang, and L. Hong, "The global responses characteristics of a rotor/stator rubbing system with dry friction effects," Journal of Sound and Vibration, vol. 330, no. 10, pp. 2150-2160, 2011.

[5] X. Han and Q. Bi, "Generation of hysteresis cycles with two and four jumps in a shape memory oscillator," Nonlinear Dynamics, vol. 72, no. 1-2, pp. 407-415, 2013.

[6] J.-P. Françoise and C. Piquet, "Hysteresis dynamics, bursting oscillations and evolution to chaotic regimes," Acta Biotheoretica, vol. 53, no. 4, pp. 381-392, 2005.

[7] V. Piccirillo, J. M. Balthazar, and B. R. Pontes Jr., "Analytical study of the nonlinear behavior of a shape memory oscillator: Part I-primary resonance and free response at low temperatures," Nonlinear Dynamics, vol. 59, no. 4, pp. 733-746, 2010.

[8] L. C. Silva, M. A. Savi, and A. Paiva, "Nonlinear dynamics of a rotordynamic nonsmooth shape memory alloy system," Journal of Sound and Vibration, vol. 332, no. 3, pp. 608-621, 2013.

[9] M. D. Bernardo, P. Kowalczyk, and A. Nordmark, "Bifurcation of dynamical systems with sliding: derivation of normal-form mappings," Physica D: Nonlinear Phenomena, vol. 170, no. 3-4, pp. 175-205, 2002.

[10] M. A. Teixeira, "Generic bifurcation in manifolds with boundary," Journal of Differential Equations, vol. 25, no. 1, pp. 65-89, 1977.

[11] Z. Sun, W. Xu, X. Yang, and T. Fang, "Effects of time delays on bifurcation and chaos in a non-autonomous system with multiple time delays," Chaos, Solitons \& Fractals, vol. 31, no. 1, pp. 39-53, 2007.

[12] Y. G. Zheng and Z. H. Wang, "The impact of delayed feedback on the pulsating oscillations of class-B lasers," International Journal of Non-linear Mechanics, vol. 45, no. 7, pp. 727-733, 2010.

[13] X. Chen, Y.-M. Kang, and Y.-X. Fu, "Switches in a genetic regulatory system under multiplicative non-Gaussian noise," Journal of Theoretical Biology, vol. 435, pp. 134-144, 2017.

[14] R. Qu, Y. Wang, G. Q. Wu, Z. D. Zhang, and Q. S. Bi, "Bursting oscillations and the mechanism with sliding bifurcations in a Filippov dynamical system," International Journal of Bifurcation and Chaos, vol. 28, no. 12, 2018.

[15] R. Rocha, J. Ruthiramoorthy, and T. Kathamuthu, "Memristive oscillator based on Chua's circuit: stability analysis and hidden dynamics," Nonlinear Dynamics, vol. 88, no. 4, pp. 2577-2587, 2017. 
[16] M. Desroches, B. Krauskopd, and H. M. Osinga, "Numerical continuation of canard orbits in slow-fast dynamical systems," Nonlinearity, vol. 23, no. 3, pp. 739-765, 2010.

[17] T. T. Hartley and F. Mossayebi, "On the control of an array Chua's systems," in Proceedings of the IEEE International Symposium on Circuits Systems, vol. 78, no. 7, pp. 1536-1539, Seattle, WA, USA, December 1995.

[18] R. Prohens, A. E. Teruel, and C. Vich, "Slow-fast n-dimensional piecewise linear differential systems," Journal of Differential Equations, vol. 260, no. 2, pp. 1865-1892, 2016.

[19] H. Simo and P. Woafo, "Bursting oscillation in electromechanical systems," Mechanics Research Communications, vol. 38, no. 8, pp. 537-541, 2011.

[20] Q. S. Bi, S. L. Li, J. Kurths, and Z. D. Zhang, "The mechanism of bursting oscillations with different codimensional bifurcations and nonlinear structures," Nonlinear Dynamics, vol. 85 , no. 2, pp. 993-1005, 2016.

[21] M. Desroches, M. Krupa, and S. Rodrigues, "Spike-adding in parabolic bursters: the role of folded-saddle canards," Physica D: Nonlinear Phenomena, vol. 331, no. 1, pp. 58-70, 2016.

[22] B. Sandstede, "Center manifolds for homoclinic solutions," Journal of Dynamics and Differential Equations, vol. 12, no. 3, pp. 449-510, 2000.

[23] H. M. Osinga, "Nonorientable manifolds in three-dimensional vector fields," International Journal of Bifurcation and Chaos, vol. 13, no. 3, pp. 553-570, 2003.

[24] J. Murdock, Normal Forms and Unfoldings for Local Dynamic Systems, Springer-Verlag, New York, NY, USA, 2003.

[25] R. I. Leine, "Bifurcations of equilibria in non-smooth continuous systems," Physica D: Nonlinear Phenomena, vol. 223, no. 1, pp. 121-137, 2006. 


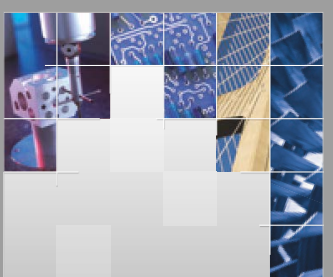

\section{Enfincering}
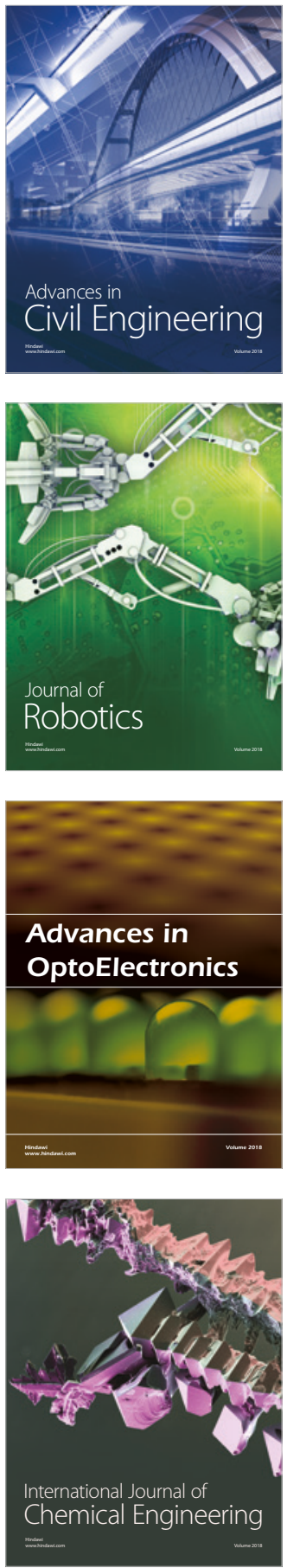

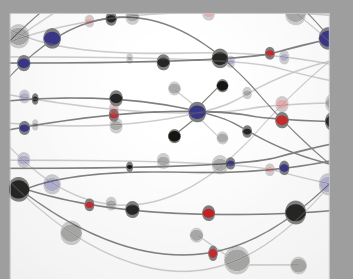

\section{Rotating \\ Machinery}

The Scientific World Journal

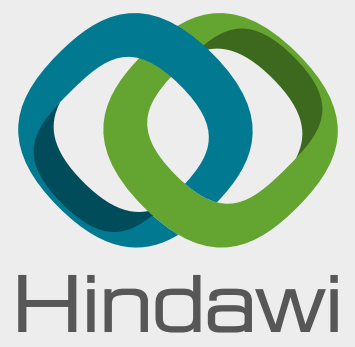

Submit your manuscripts at

www.hindawi.com
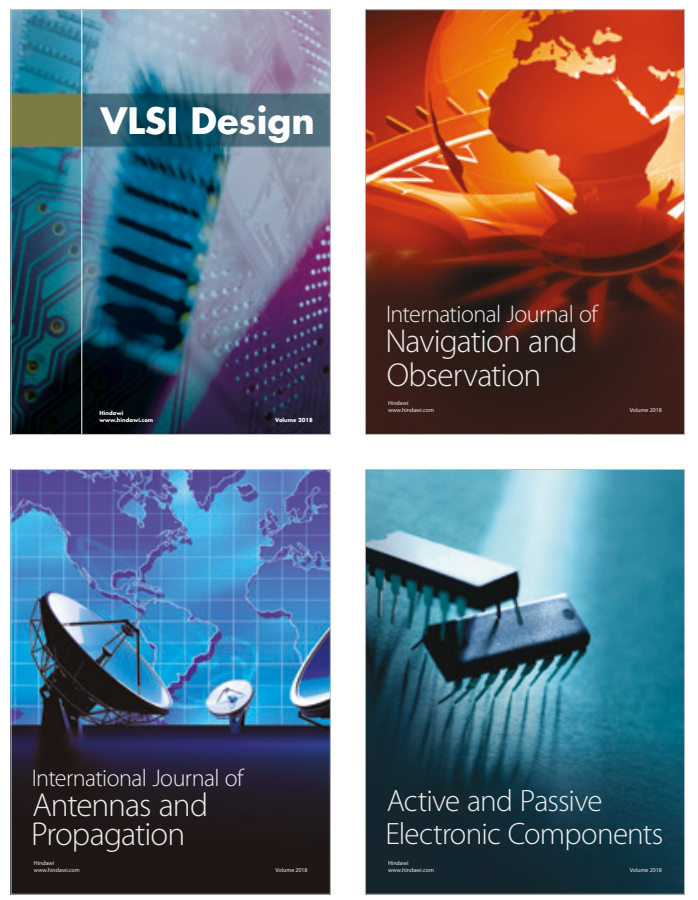
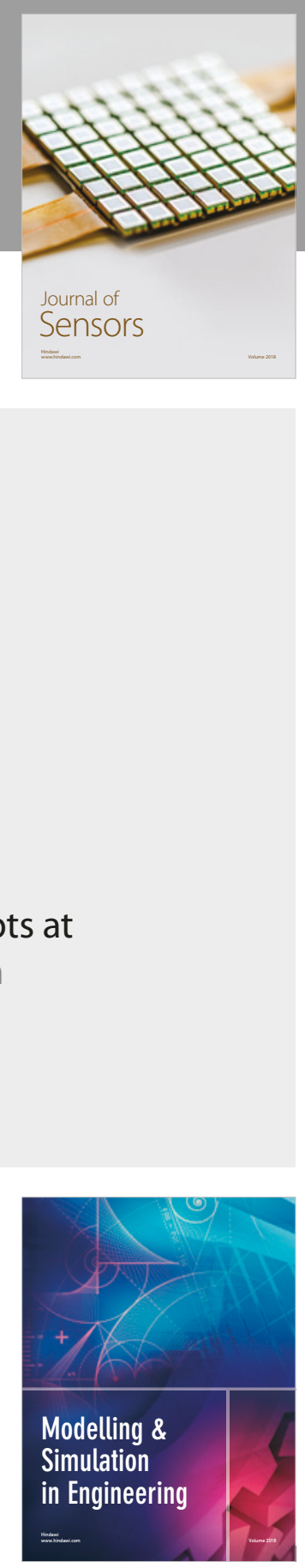

\section{Advances \\ Multimedia}
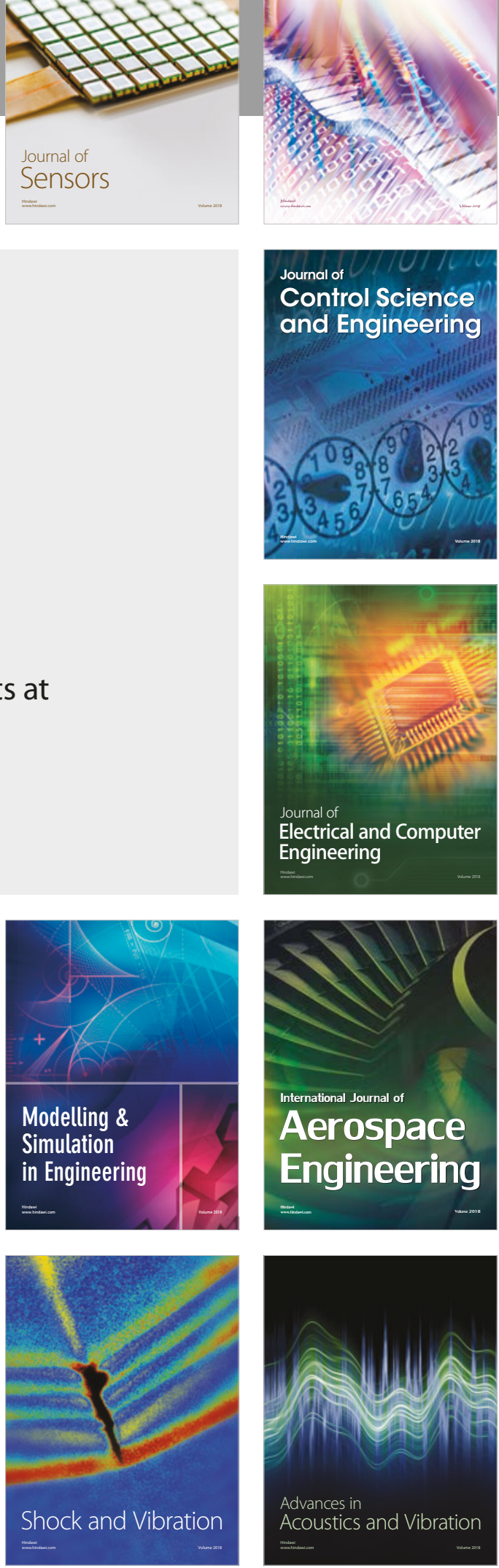